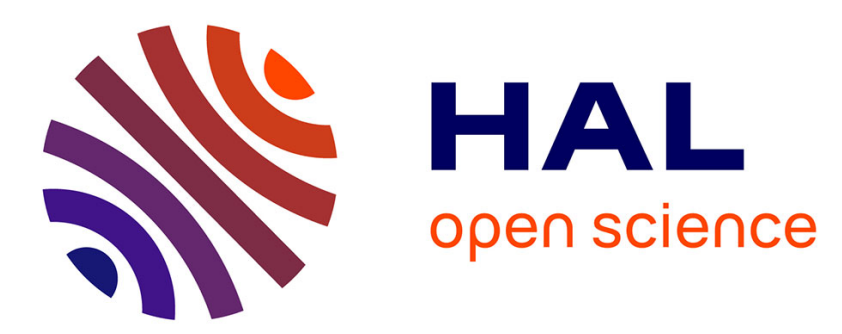

\title{
Predicting blocking effects in the spatial domain using a learning approach
}

\author{
Aladine Chetouani, Ghiles Mostafaoui, Azeddine Beghdadi
}

\section{To cite this version:}

Aladine Chetouani, Ghiles Mostafaoui, Azeddine Beghdadi. Predicting blocking effects in the spatial domain using a learning approach. ICETE/SIGMAP08, Jul 2008, porto, Portugal. hal-00362792v2

\section{HAL Id: hal-00362792 \\ https://hal.science/hal-00362792v2}

Submitted on 2 Feb 2011

HAL is a multi-disciplinary open access archive for the deposit and dissemination of scientific research documents, whether they are published or not. The documents may come from teaching and research institutions in France or abroad, or from public or private research centers.
L'archive ouverte pluridisciplinaire HAL, est destinée au dépôt et à la diffusion de documents scientifiques de niveau recherche, publiés ou non, émanant des établissements d'enseignement et de recherche français ou étrangers, des laboratoires publics ou privés. 


\title{
Predicting blocking effects in the spatial domain using a learning approach
}

\author{
Aladine Chetouani, Ghiles Mostafaoui and Azeddine Beghdadi \\ Laboratory L2TI, Institute of Galilee, University Paris XIII, France \\ aladine.chetouani@12ti.univ-paris13.fr,ghiles.mostafaoui@12ti.univ-paris13.fr \\ azeddine.beghdadi@12ti.univ-paris13.fr
}

\begin{abstract}
A new method for predicting blocking effect in the spatial domain is proposed. This method aims at estimating the appearance of blocking artefacts in the original image prior to compression for a given bit rate and a given compression technique. The basic idea is to use a training process in order to compute a visibility measure. A weighting function of the blocking effects is then derived from this training process performed on a database. The proposed method is objectively and subjectively evaluated on various actual images. The obtained results confirm the efficiency of the proposed method in predicting blocking effect.
\end{abstract}

\section{Introduction}

Block-based image processing approaches are systematically applied to account for the non stationarity of the image signal and the computational constraints. The other motivation behind the development of block-based image treatments is to make them appropriate for real-time application and their possible implementation on parallel architectures. However, block-based methods are prone to artefacts, called blocking effect, that may affect the image quality and limit the efficiency of some image processing techniques. This artefact is the most known annoying image distortion. The efficiency limitation of many compression methods, such as BTC (Block Truncation Coding) technique [1] or VQ (Vector Quantization) coding [2], is essentially due to blocking effect. Many improvements have been proposed in order to reduce this effect. But at our knowledge, the design of formalized procedures allowing to control this effect is still missing.

Here, we focus our study on block-based compression methods and especially those using Discrete Cosine Transform (DCT). This method has been widely used in image and video compression standards such as JPEG and MPEG2. For low bit rate, these block-based coding methods produce a noticeable blocking effect, in the reconstructed image. This is mainly due to the fact that the blocks are transformed and quantized independently. This annoying artefact appears at the block frontiers as artificial horizontal and vertical contours. The visibility of this blocking effect depends highly on the spatial intensity distribution in the image. Moreover, the Human Visual System (HVS) increases the perceived contrast between two adjacent regions. This is called the Mach effect [3]. Therefore, the visibility of the blocking effect is more amplified due to the pronounced horizontal and vertical sensitivity of the HVS.

Blocking effect has been widely studied and many had hoc methods for estimating and reducing this artefact have been proposed in the literature [4]-[9]. In [4], a blind method for estimating the blocking effect in the frequency domain is proposed. The absolute differences along each row are computed and rearranged into vector to form a 1-D signal. The power spectrum is then derived from this $1 \mathrm{D}$ signal. The absolute difference of the power spectrum is used as a global visibility measure of the blocking effect.

It worth to noticing that blind approaches are more appealing than full reference approaches [5]-[8]. Indeed, in practice the original signal is not available. In [5],[8], the blocking artefacts are modelled as 2D signals in the DCTcoded images. By taking into account some HVS properties, the local contrast in the vicinity of the inter-block boundary is used as an estimate of the blocking effect. In [5], the visibility measure of the blocking effect is expressed in terms of the sum and difference of two adjacent blocks by taking into account some properties of the Human Visual System.

In [9], an iterative algorithm is applied for reducing the blocking effect artefact in the block transform-coded images by using a minimum mean square error filter in the wavelet domain. Another similar method based on image restoration approach has been proposed [10]. A blocking effect visibility measure based on the local contrast is used to control the iterative process.

In [11], a new technique based on a frequency analysis is proposed for detecting blocking effects. The artefacts are modelled as 2-D step function between the neighbouring blocks. The presence of the blocking artefacts is detected by using block activity signal based on HVS and block statistics.

Several other interesting methods [12]-[16], dealing with blocking effects have been also developed. However, Most of these studies aim to detect or remove the blocking effects on the compressed images. 
Here, we propose a different approach which allows to predict the visibility of the blocking effects on images prior to compression. The main objective of this method is to prevent the degradations due to compression during exchange, sharing and visualisation (video streaming, VOD). More precisely, for a given bit rate and a block-based compression method, we associate to each region in the original non compressed image a weight related to the visibility of the blocking effect. This weighting could be interpreted as the probability of appearance of blocking effect. The paper is organized as follows. Section 2 presents the motivations and describes in details the weighting procedure. Section 3 is dedicated to the results and the performance evaluation of our method. The last section contains the conclusion and perspectives.

\section{Motivation and method}

The continuing development of high resolution imagery technology leads to higher bit rates because of the increase in both spatial resolution and intensity range. Much research on block-based lossy image compression is still needed. However, lossy compression at low bit rate may produce some annoying artefacts limiting thus their efficiency. Here, we focus the study on blocking effects. One of the main issues related to image compression is how to control these artefacts. One way to achieve this goal is first to predict this structured distortion and then to propose a solution for reducing it. Inspired by the fact that human observer is able to detect and recognize blocking effect, even in the absence of the original image, we propose a new approach based on a learning process. Because, we believe that this process is undoubtedly related to some a priori knowledge recorded in the brain through visual observation. The approach use here is based on a training offline process. The main idea is to compute a weighting function which assigns to each pixel a weight that could be interpreted as a prediction probability of the appearance of the local blocking effect.

The main idea developed here is to study the relation between the appearance of the blocking effects and the pixels neighbourhoods in the non-compressed image. Therefore, we perform a learning offline process on a database containing various grey-level and color images. Some samples from this database are shown in fig. 1 . The whole weighting process is summarized in fig. 2.

\subsection{Learning process}

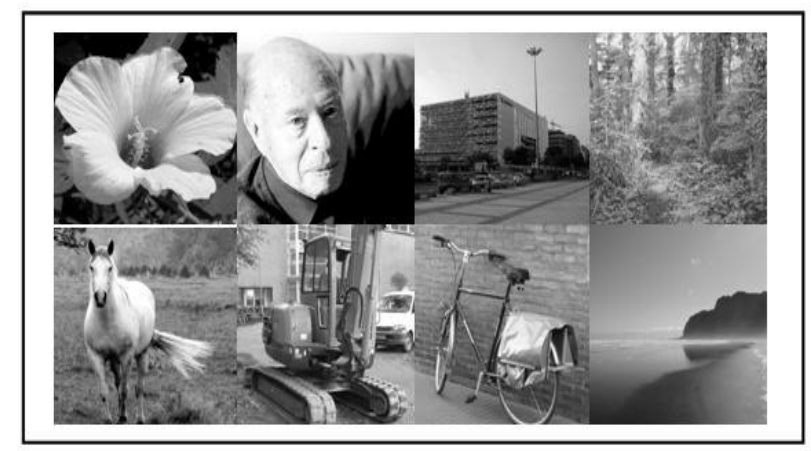

Figure 1: Samples from CCN image database

The learning process is applied on a database of 211 different real images (from F. C. Donders Centre for Cognitive Neuroimaging database, see figure 1). These images contain various kinds of textures with different roughness and regions with different intensity distribution and uniform regions.

First, we analyze the spatial distribution of the pixels before extracting some local characteristics from these images. Indeed, the appearance of a blocking effect in an image area depends highly on the local descriptors such as color, homogeneity, gradient etc. These local characteristics could be expressed in the transformed domain such as Wavelet Transform or DCT. To make the method independent of compression method, we use the local variance which is a simple statistic measure representing the pixels neighbourhoods homogeneity.

For each image $f$ taken from the learning database, we compute the corresponding local variance image $V$ (see fig. 3.b).

Once the local variance image computed, we analyse the compression effect in terms of blocking appearance. To do this, we have to detect the blocking effects on the compressed images. Let us define:

- $g_{q}$ the compressed images of an original image $f$ of the database where $q$ represents the different quality factors ( $q \in[1,100]$ for JPEG compression).

- $g_{q}^{\prime}$ the gradient absolute values images of $g_{q}$. 


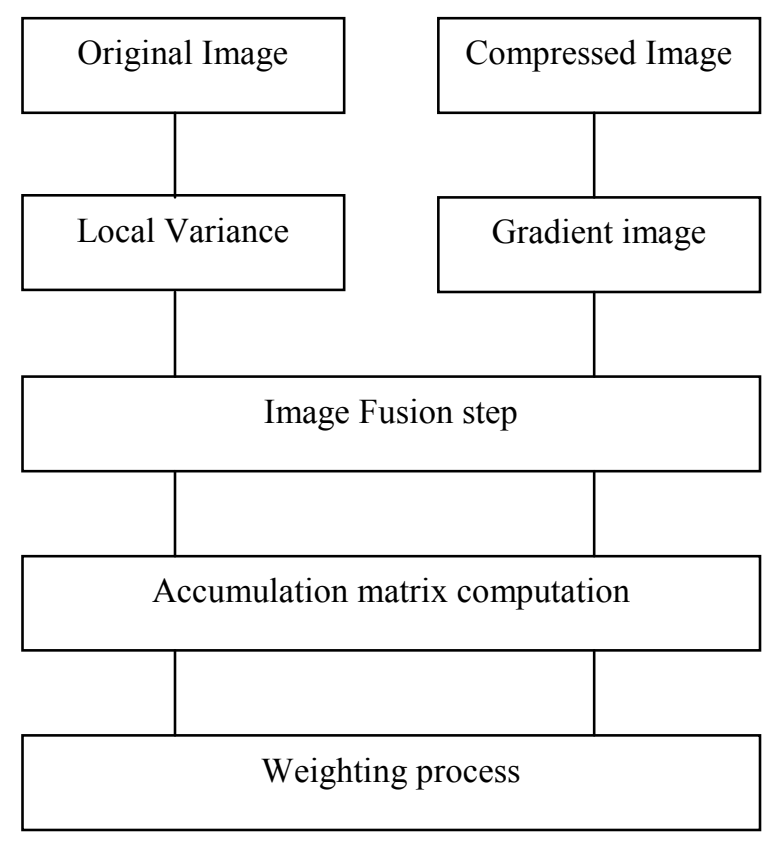

Figure 2: Synoptic

Depending on the bit rate, the blocking effect tends to create large uniform zones where the gradient is null. The blocking effects on the compressed images $g_{q}$ could be then detected by analyzing the signal $g_{q}^{\prime}$.

This first simple process gives coarse detection of blocking effect (see fig. 3.c). We will show that by increasing the size of the database, used in the learning process, the blocking effect detection could be improved.

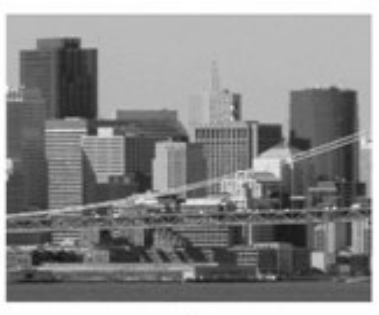

a)

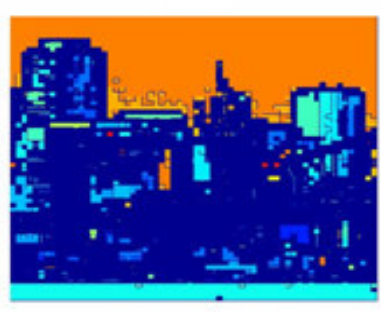

c)

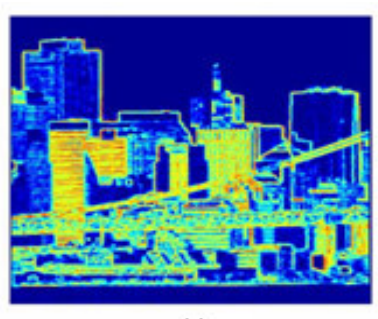

b)

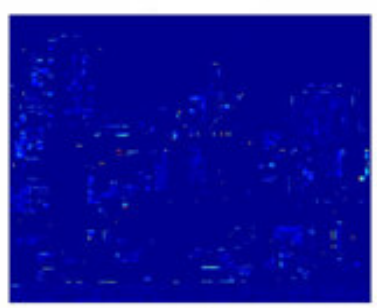

d)
Figure 3: a) Original image, b) Local variances image, c) Labelled regions with null gradients in the compressed image, d) Local variances of pixels with null gradients in the compressed image.
Here, we use a cumulative learning method based on a voting process. A table of accumulation representing the statistics of the appearance of blocking effect in the compressed images ( at different quality factors) is computed. This vote table is a 2D array denoted $H(v, q)$ where $v$ is the possible values of the variance $(v \in[0,255])$ and $q$ the compression quality factor $(q \in[1,100])$.

Let $\left\{f^{i}, i \in[1, N]\right\}$ be the set of database images, $V^{i}, g_{q}^{i}$ and $g_{q}^{\prime i}(q \in[1,100])$ the corresponding images obtained as explained above.

For each pixel $p_{j}^{i}\left(x_{j}^{i}, y_{j}^{i}\right)$ of an image $f^{i}$ we define an influence function for each couple $(v, q)$. This function can be written as:

$C c_{j}^{i}(v, q)=\left\{\begin{array}{cr}1 \text { if } v=V^{i}\left(x_{j}^{i} y_{j}^{i}\right) \text { and } g_{q}^{\prime i}=0 \\ 0 & \text { else }\end{array}\right.$

This expression means that a pixel will have a positive influence on a cell $(v, q)$ of $H(v, q)$ only if its local variance corresponds to $v$ and its gradient absolute value on the compressed image $\left(g_{q}^{i}\right)$ with $q$ as quality factor is null (pixel probably belonging to a blocking effect on $g_{q}^{i}$ ).

After computing the influence functions for all the pixels $\left(p_{j}^{i}\left(x_{j}^{i}, y_{j}^{i}\right)\right)$ of all the images $f^{i}$ of the database, we can define the value of a cell $(v, q)$ of the accumulation table as follows :

$$
H(v, q)=\sum_{i} \sum_{j} c_{j}^{i}(v, q)
$$

Fig. 4 displays a part of the accumulation matrix corresponding to variances between 0 and 16 for a better visibility.

This table contains the statistical information about the pixel neighbourhood and the corresponding degree of blocking effect appearance. It contains all the relevant local characteristics and compression factors. 


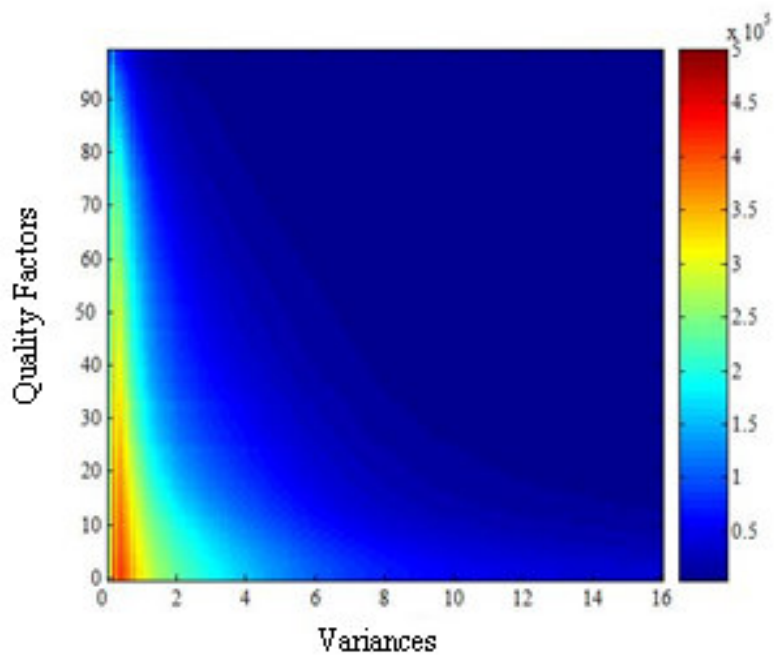

Figure 4: The accumulation matrix

Fig. 4 clearly shows the coherence of the statistics. In fact, one can notice that less the variance is and less the quality factor is (high compression ratio), higher is the probability of appearance of a blocking effect. The errors due to approximations are completely cancelled by the large mass of correct accumulations.

\subsection{The weighting process}

The obtained voting matrix is used for predicting blocking effect. A weighting function, representing the probability of appearance of a blocking effect on a pixel neighbourhood, is then derived from this table. For each factor quality value, a simple weighting function is to consider the row of the matrix $H$ given by:

$$
w_{q}(v)=H(v, q) \quad v \in[0,255]
$$

The matrix $H$ is constructed from the image database. It could be noticed that we have no guarantee that all the possible values of the local variance will be represented. Furthermore, for natural images the homogeneous regions are predominant. Low local variance values are then more often encountered than high variances. This leads to a lack of accumulations for some cells in the voting table. To cope with this drawback, we apply a polynomial interpolation to the weight function defined in equation 3 . After a normalization step, we obtain the final weight functions shown in fig. 5.

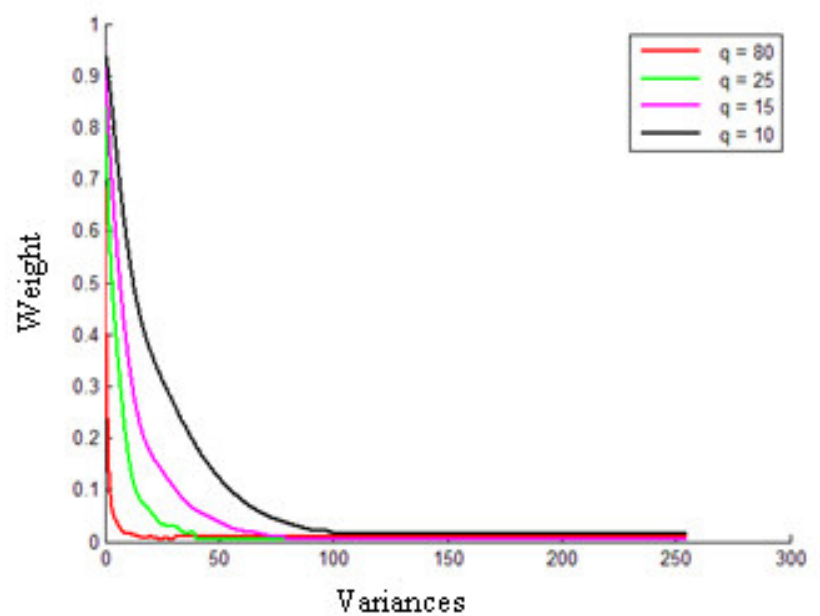

Figure 5: The weighting function for different quality factors.

Here, we can also notice the coherence of the results related to the fact that for a given local variance value, lower the quality factor is (high compression ratio), higher the weights are (increase of the appearance probability of blocking effects).

\section{Experimental results}

To evaluate the efficiency of the proposed method in predicting blocking effect, we use both objective and subjective assessment. In the following, we describe the two strategies in details. Since, the subjective evaluation of perceptual distortion measure is the most accepted approach, we evaluate the objective measure in terms of correlation with the MOS obtained through subjective tests.

\subsection{Objective test}

To test the efficiency of the proposed measure, various experimental tests are realized over 200 natural images different from those of the learning database and with variable bit rate. The experimental procedure is quite simple and does not require the compressed images. We use only the uncompressed images. Let $f$ be an original test image and $V$ its corresponding local variance image. The probability of a pixel $(x, y)$ to belong to a blocking effect area with a given quality factor $q$ of compression (here JPEG) is :

$$
w_{q}(v) \text { with } v=V(x, y)
$$


$w_{q}$ representing the weight function obtained from the learning process. This makes the method very fast. In fact, for implementation, the weight functions (obtained with the offline learning) can be seen as simple Look Up Table. The prediction procedure is then reduced to computing the local variances.

Fig. 6, gives an example of the predicted weight images obtained for a natural test image. Fig. 6.a is the original test image. And we choose three quality factor $\mathrm{q}=49,17,4$ and the predicted weight image are represented in the fig. 6.b, 6.c and 6.d respectively. Let us define that the red and blue regions correspond to the high and low weights respectively.

The obtained results show the gradual increase of the weights when the quality factor is reduced and efficiently express the fact that homogeneous regions are affected more quickly (with the reduction of $q$ ) by the blocking effect than the textured ones. The same observations are shown with different content images. An over result is presented in the fig. 8. We present the original test image fig. 8.a, the compressed image with a quality factor equal to 10 in fig $8 . b$ and the predicted weight image in fig. 8.c. Effectively, the high weights are affected at the regions that are more affected by the compression artefacts.

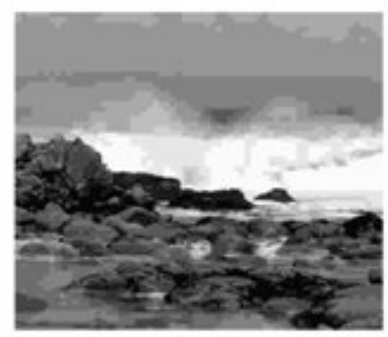

a)

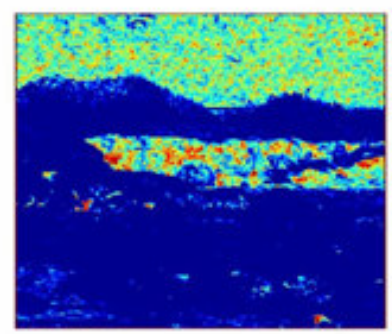

c)

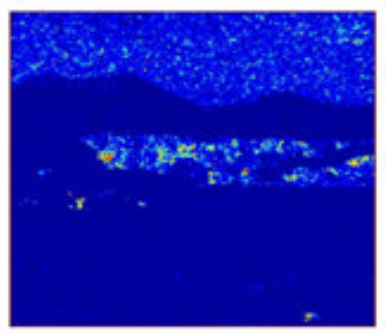

b)

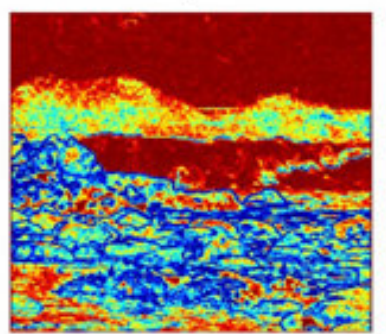

d)
Figure 6: Blocking effect prediction. a) Original image, b)-d) : Blocking effect visibility map for $\mathrm{q}=49, \mathrm{q}=17$ and $\mathrm{q}=4$, respectively

\subsection{Subjective tests}

Subjective evaluation of image quality is still the most accepted solution. Unfortunately, it necessitates the use of several procedures, which have been formalized by the ITU recommendation [17]. These procedures are complex, time consuming, and nondeterministic. It should be also noticed that perfect correlation with the HVS could never be achieved due to the natural variations in the subjective quality evaluation. In our experiments, we performed subjective tests with ten observers. We present to each observer, various images with different quality factor values $q$. The observers are asked to identify for each compression ratio (quality factor) and for each image of the database, the appearance of blocking effects.

Fig. 7 shows the Mean Opinion Score (MOS) for each image (white line) used in the subjective tests. This MOS corresponds to a quality factor value for which the blocking effect starts to be visible. For each quality factor $(q \in[1,100])$ and for each test image, we compute the corresponding weight using the weighting function. The obtained results show that the mean observer starts to see blocking effects on the compressed images at ratio corresponding to prediction probabilities up to 0.4 .

\section{Quality Factor for which the mean observer start to see a blocking effect \\ Predicted weights}

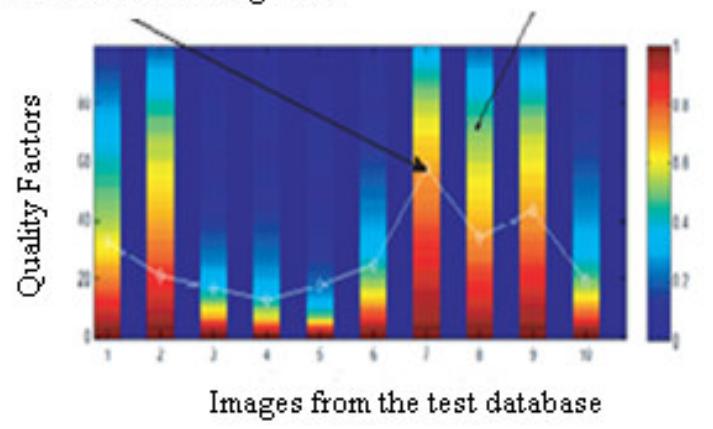

Figure 7: Objective vs subjective quality measure

\section{Conclusion and perspectives}

A simple and efficient method for predicting blocking effects on the original non-compressed image based on a local image analysis and a training scheme is proposed. The obtained results show that the proposed method is efficient in predicting blocking effect and show good correlation with subjective evaluation. This predictive scheme could be used as a blind image quality control system prior to compression in order to achieve the trade-off between bit rate and perceptual image quality.

As perspective, we are planning to introduce a masking model in the method to make it more adaptive to image signal activity and HVS limitations. This predictive method could be extended to other block-based image compression techniques. 


\section{References}

[1] E.J. Delp and O.R. Mitchel, "Image compression using block truncation coding". IEEE transactions on Communications, No 27 pp 1135-1142, 1979.

[2] R. M. Gray, "Vector Quantization", IEEE ASSP Magazine, 1, N², pp.4-29, 1984.

[3] W.T. Welford,"The visual Mach effect". Physics Education, Volume 3, Issue 2, pp. 83-84, 1968.

[4] Z. Wang, A.C. Bovik and B.L. Evans, "Blind measurement of blocking artifacts in images". IEEE International Conference on Image Processing, vol. 3, pp. 981-984, Sept. 2000.

[5] A.C. Bovik and S. Liu, "DCT-domain blind measurement of blocking artifacts in DCT-coded images". Proc. IEEE International Conference on Acoustics, Speech, and Signal Processing, May 7-11, 2001.

[6] T.K. Kim, J.K. Paik, C.S. Won, Y. Choe, J. Jeong and J.Y. Nam, "Blocking effect reduction of compressed images using classification-based constrained optimization". Signal Processing: Image Communication, Volume 15, Issue 10, pp. 869-877, August 2000.

[7] C.C Chang, C.S. Chan and C.S. Teng, "Removing blocking effects using an artificial neural network". Signal Processing, Volume 86, Issue 9, pp. 2381-2387, September 2006.

[8] F.X. Coudoux, M.G. Gazalet and P. Corlay, "Reduction of blocking effect in DCT-coded images based on a visual perception criterion". Signal Processing: Image Communication, Volume 11, Issue 3, pp. 179-186, January 1998.

[9] I.H. Jang, N.C. Kim and H.J. So, "Iterative blocking artifact reduction using a minimum mean square error filter in wavelet domain". Signal Processing 83(12), pp. 2607-2619, 2003.

[10] M. Luong, A. Beghdadi, W. Souidene and A. Le Négrate, "Coding artefact reduction using adaptive posttreatment", IEEE ISSPA, Volume 1, pp. 347-350, 2005.

[11] S. Singh, V. Kumar and H.K. Verma, "Reduction of blocking artifacts in JPEG compressed images". Digital Signal Processing, Volume 17, Issue 1, pp. 225-243, January 2007.

[12] B. Zeng, "Reduction of blocking effect in DCTcoded images using zero-masking techniques", Signal Processing, Volume 79, Issue 2, pp. 205-211, December 1999.
[13] Y.L. Lee, H.C. Kim and H.W. Park, "Blocking effect reduction of JPEG images by signal adaptative filtering". Image Processing, IEEE Transactions on Volume 7, Issue 2, pp. 229 - 234, Feb 1998.

[14] R. Castagno, S. Marsi and G. Ramponi, "A simple algorithm for the reduction of blocking artifacts in images and its implementation". IEEE Trans. on Consumer Electronics, vol. 44, no.3, Aug. 1998.

[15] S.S.O. Choy, Y.H. Chan and W.C. Siu, "Reduction of block-transform image coding artifacts by using local statistics tranform coefficients". IEEE. Signal Processing Letters, vol. 4, no. 1, pp. 5-7, Jan. 1997.

[16] G. Qiu, "MLP for adaptative postprocessing blockcoded images". IEEE Trans. Circuits and Systems for Video Technology, No. 8, pp. 1450-1454, December 2000.

[17] CCIR, "Method for subjectives assessment of the quality of television pictures", Recommendation 500-4, 19901994.

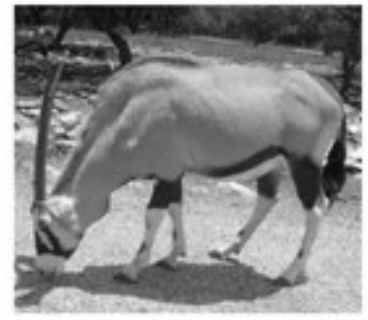

a)

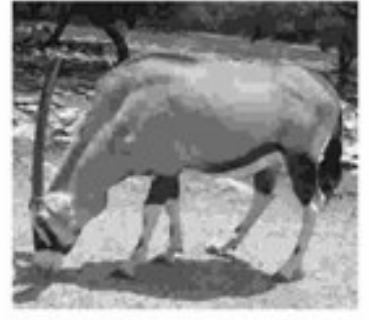

b)

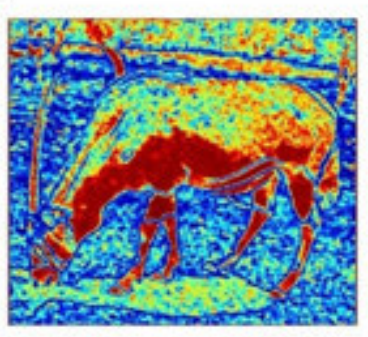

c)
Figure 8: Blocking effect prediction results: a) Original image, b) Compressed image for $q=10$, c) Blocking effect visibility map. 